\title{
[editorial]
}

\section{El efecto placebo y el dolor}

\section{Darío Scublinsky}

Doctor en Medicina. Médico Reumatólogo.

Primera Cátedra de Farmacología, Facultad de Medicina, UBA.

$\mathrm{Al}$ administrar un medicamento se generan una serie de efectos independientes de la acción farmacológica del principio activo. Estos efectos suelen ser de naturaleza biológica (mecanismo de acción de la droga) o psicológica. Si es un medicamento inyectable despertaremos en el paciente dolor, cierto grado variable de inquietud, independientemente de la droga administrada, aunque también una expectativa de que "es un medicamento fuerte". Por otro lado, en el paciente aparecerán emociones como la confianza, esperanza, mayor tranquilidad, o temor, ansiedad, inquietud.

Relataba el Profesor Tessler': Por ejemplo, cuando llegaba a casa nuestro médico, luego de saludar a todos, y por mal que fuera el aspecto del enfermo, enseguida decía: "Pero, ¿quién es el enfermo?" con lo cual el aludido se sentía ya mucho mejor.

El efecto psicológico del acto médico, aunque no poseía valor desde el punto de vista químico, mejoraba al paciente al sentirse cuidado. Cuando este acto se transmitió a través de un medicamento se lo llamó "placebo", del latín placeo (te complaceré). Entonces, al resultado buscado administrando un placebo, se lo llamó "efecto placebo". Este efecto es hoy concebido como los cambios psicológicos directos y las modificaciones biológicas que se producen como consecuencia del uso de un placebo ${ }^{2}$.

El placebo no medicamentoso es tan antiguo como la cultura. La historia de la Medicina está plagada de dichos placebos: punciones, cataplasmas, ventosas, descargas eléctricas, etc. El placebo medicamentoso nace con la farmacología moderna, y el placebo como el mejor grupo de comparación nace con la farmacología clínica a mediados del siglo XX. Es así que el placebo en investigación surgió de la necesidad de evaluar si los cambios producidos en un enfermo eran generados por el medicamento en cuestión o por la propia evolución natural de la enfermedad. Hoy en día, por cuestiones éticas y por conocerse la evolución natural de la mayor parte de las enfermedades, la utilización de un placebo implica valorar los riesgos y beneficios que puede tener el paciente con dicha terapéutica, siendo su uso habitual y aceptado en el marco de la investigación farmacológica.

La neurobiología del placebo nació en 1978, cuando se demostró que la analgesia del placebo podía ser bloqueada por un antagonista opioide como la naloxona en una dosis como para bloquear el efecto de $8 \mathrm{mg}$ de morfina. Luego se detectó que el placebo induce la activación de la neurotransmisión opioide, que fue identificada en el área cingulada anterior y en las cortezas orbitofrontal e insular, el núcleo accumbens, amígdala y sustancia gris periacueductal. También se detectó activación dopaminérgica en los ganglios basales, incluyendo el núcleo accumbens. Es así que la actividad dopaminérgica y opioide fue asociada con la anticipación analgésica y la eficacia del placebo. En especial, la liberación de dopamina en el núcleo accumbens fue asociada con la "expectativa de analgesia". Las altas tasas de respuesta al placebo fueron asociadas con mayor actividad de dopamina (sobre receptores D2/D3) y opioides (sobre receptores mu) en el núcleo accumbens, mientras que efectos adversos por el uso del placebo (nocebo) fueron asociados con la desactivación de la liberación de dopamina y opioides ${ }^{3,4}$. De tal forma que los efectos placebo y nocebo fueron asociados con respuestas opuestas en la neurotransmisión dopaminérgica y opioidea. También una hiperalgesia con el uso de placebo fue vinculada a hiperactividad del eje hipotálamo-pituitario-adrenal y las concentraciones plasmáticas de cortisol. Este efecto fue antagonizado por diazepam por lo cual la ansiedad jugaría un rol central en dicho efecto ${ }^{5}$.

De 69 estudios clínicos ciegos randomizados que compararon diferentes clases de drogas utilizadas en la migraña y placebos, en los ensayos que estudiaron AINEs, los pacientes asignados al grupo placebo presentaron con gran frecuencia náuseas, vómitos y otros trastornos gastrointestinales, efectos adversos habitualmente atribuidos a los AINEs $s^{6}$.

En conclusión, placebo no es sinónimo de efecto psicológico del medicamento, como solemos decir los médicos, sino que en muchos casos existe también un efecto biológico, íntimamente vinculado a los factores cognitivos del paciente que no debemos menospreciar en la práctica clínica o en investigación.

\section{Bibliografía}

1. Tessler J. Farmacologia Básica. 1997.

2. Benedetti F. Placebo analgesia. Neurological sciences: official journal of the Italian Neurological Society and of the Italian Society of Clinical Neurophysiology. May 2006;27 Suppl 2:S100-102.

3. Barsky AJ, Saintfort R, Rogers MP, Borus JF. Nonspecific medication side effects and the nocebo phenomenon. JAMA : the journal of the American Medical Association. Feb 6 2002;287(5):622-627.

4. Bartels DJ, van Laarhoven AI, Haverkamp EA, et al. Role of conditioning and verbal suggestion in placebo and nocebo effects on itch. PloS one. 2014;9(3):e91727.

5. Zubieta JK, Stohler CS. Neurobiological mechanisms of placebo responses. Annals of the New York Academy of Sciences. Mar 2009;1156:198-210.

6. Amanzio M, Corazzini LL, Vase L, Benedetti F. A systematic review of adverse events in placebo groups of anti-migraine clinical trials. Pain. Dec 2009;146(3):261269.

\section{Correspondencia}

E-mail: darioscublinsky@yahoo.com.ar 